\title{
FLAT ORLICZ SPACES
}

\author{
A. J. PACH, M. A. SMITH $^{1}$ AND B. TURETT ${ }^{2}$
}

\begin{abstract}
Let $(\Omega, \Sigma, \mu)$ be a finite measure space that is not purely atomic and let $\Phi$ be a Young function. It is shown that the Orlicz space $L^{\oplus}(\mu)$ is flat if and only if either $L^{\Phi}(\mu)$ contains an isometric copy of $l^{\infty}$ or $L^{\Phi}(\mu)$ is isometric to $L^{1}(\mu)$.
\end{abstract}

A Banach space $X$ is called flat if there exists a curve on the surface of the unit ball of $X$ with antipodal endpoints and length two. Although one's initial (finite dimensional) reaction to this definition is to question the existence of such spaces, Harrell and Karlovitz ([1] and [2]) have shown that some of the classical Banach spaces are flat; in [2], they showed that $C[0,1]$ is flat. This result was later generalized by Nyikos and Schäffer [4] who characterized flat $C_{\sigma}(K)$ spaces; in particular, they noted that every infinite dimensional $L^{\infty}(\mu)$ is flat. Since Harrell and Karlovitz [2] proved that no flat Banach space is reflexive, the only Lebesgue spaces other than the $L^{\infty}(\mu)$ spaces that could be flat are the infinite dimensional $L^{1}(\mu)$ spaces. In this direction, Harrell and Karlovitz [1] noted that $L^{1}(\lambda)$ where $\lambda$ is Lebesgue measure on $[0,1]$ is flat while $l^{1}$ is not. Schäffer [6] then showed that the Lebesgue space $L^{1}(\mu)$ is flat if and only if the measure space $(\Omega, \Sigma, \mu)$ is not purely atomic.

In this paper, flat Orlicz spaces will be characterized in the class of all Orlicz spaces over finite measure spaces that are not purely atomic. Specifically, it will be shown that if $(\Omega, \Sigma, \mu)$ is a finite measure space that is not purely atomic, an Orlicz space $L^{\Phi}(\mu)$ is flat precisely when $L^{\Phi}(\mu)$ contains an isometric copy of $l^{\infty}$ or when $L^{\Phi}(\mu)$ is isometric to $L^{1}(\mu)$. Thus, by widening the view to Orlicz spaces rather than just Lebesgue spaces, essentially no additional flat Banach spaces are found.

The terminology used in this paper is relatively standard but it is quickly recalled now for the sake of definiteness. A measure space $(\Omega, \Sigma, \mu)$ is said to be not purely atomic if there exists a set $S$ in $\Sigma$ such that $0<\mu(S)<\infty$ and $S$ contains no atoms. The function $\Phi: \mathbf{R} \rightarrow \mathbf{R}_{e}$ will always denote a Young function; that is, $\Phi$ is a nonnegative, not identically zero, even, convex, extended real-valued function with $\Phi(0)=0$ and $\Phi(x)<\infty$ for some $x \neq 0$. The Orlicz space $L^{\Phi}(\mu)$ is then defined as the set of equivalence classes of $\mu$-measurable functions $f: \Omega \rightarrow \mathbf{R}$ such that $\int \Phi(k f) d \mu<\infty$ for some $k>0$. Under the norm

$$
\|f\|_{\Phi}=\inf \left\{r>0: \int \Phi(f / r) d \mu<1\right\},
$$

Received by the editors May 8, 1980; presented to the Society, January 6, 1980.

1980 Mathematics Subject Classification. Primary 46B20, 46E30.

Key words and phrases. Orlicz space, flat Banach space.

'Supported in part by National Science Foundation Grant MCS 78-02265.

${ }^{2}$ Supported in part by an Oakland University Research Fellowship.

(C) 1981 American Mathematical Society 0002-9939/81/0000-0155/\$01.75 
the Orlicz space $L^{\Phi}(\mu)$ is a Banach space. Recall that a Young function $\Phi$ is said to satisfy a $\Delta_{2}$-condition for large values of $x$ if there exist constants $M>0$ and $x^{\prime}>0$ such that $\Phi\left(x^{\prime}\right)<\infty$ and $\Phi(2 x)<M \Phi(x)$ if $x>x^{\prime}$.

Given a flat Banach space $X$, a simple curve $g:[0,4] \rightarrow X$ can be defined such that $\|g(s)\|=1$ and $g(s+2)=-g(s)$ for $s$ in $[0,2]$, and $\|g(s)-g(t)\|=|s-t|$ for $s$ and $t$ in [0,2]. Such a simple, closed, symmetric curve is called a girth curve of $X$ (see [5]). Since this paper deals with flat Orlicz function spaces, given a girth curve $g:[0,4] \rightarrow L^{\Phi}(\mu)$, write $g_{s}$ for $g(s)$ and hence write $g_{s}(\omega)$ for $g(s)(\omega)$ where $s$ is in $[0,4]$ and $\omega$ is in $\Omega$.

The aforementioned characterization of flat Orlicz spaces is now given.

TheOREM. Let $(\Omega, \Sigma, \mu)$ be a finite measure space that is not purely atomic and let $\Phi$ be a Young function. The following assertions are equivalent:

(a) The Orlicz space $L^{\Phi}(\mu)$ is flat.

(b) Either $\Phi$ fails to satisfy a $\Delta_{2}$-condition for large values of $x$ or $\Phi(x)=k|x|$ for some $k>0$.

(c) Either $L^{\Phi}(\mu)$ contains a closed subspace isometric to $l^{\infty}$ or $L^{\Phi}(\mu)$ is isometric to $L^{1}(\mu)$.

(d) The Orlicz space $L^{\Phi}(\mu)$ contains a closed subspace isometric to $L^{1}[0,1]$.

Proof. That (b) implies (c) follows immediately from Theorem 4 of [7]. That (c) implies (d) follows since every separable Banach space is isometric to some closed subspace of $l^{\infty}$ and since $L^{1}(\mu)$ contains a closed subspace isometric to $L^{1}[0,1]$ whenever $(\Omega, \Sigma, \mu)$ is not purely atomic (see [7, Lemma 2]). That statement (d) implies (a) is trivial since $L^{1}[0,1]$ is flat.

It remains to be shown that (a) implies (b). Suppose $L^{\Phi}(\mu)$ is flat and $\Phi$ satisfies a $\Delta_{2}$-condition for large values of $x$. Let $g$ be a girth curve of $L^{\Phi}(\mu)$. Let $x_{0}=\sup \{x \in \mathbf{R}: \Phi(x)<1 /(2 \mu(\Omega))\}$ and let $D=\left\{\omega \in \Omega:\left|g_{0}(\omega)\right|>x_{0}\right\}$. Then $\mu(D)>0$ since otherwise $\Phi\left(g_{0}\right)<1 /(2 \mu(\Omega))$ almost everywhere, contradicting the fact that $\int \Phi\left(g_{0}\right) d \mu=1$. (Under the assumption on $\Phi, \int \Phi(f) d \mu=1$ for each norm-one $f$ in $L^{\Phi}(\mu)[3$, p. 78].)

Let $0<s<2$. Observe that

$$
g_{0}=\frac{s}{2} \frac{g_{0}-g_{s}}{s}+\frac{2-s}{2} \frac{g_{s}-g_{2}}{2-s}
$$

and hence, by the convexity of $\Phi$,

$$
\Phi\left(g_{0}\right)<\frac{s}{2} \Phi\left(\frac{g_{0}-g_{s}}{s}\right)+\frac{2-s}{2} \Phi\left(\frac{g_{s}-g_{2}}{2-s}\right) .
$$

Since $g_{0},\left(g_{0}-g_{s}\right) / s$, and $\left(g_{s}-g_{2}\right) /(2-s)$ are norm-one functions in $L^{\Phi}(\mu)$, it follows, from [3, p. 78] again, that the integrals of the left-hand and right-hand sides of the preceding inequality are one. Therefore

$$
\Phi\left(g_{0}\right)=\frac{s}{2} \Phi\left(\frac{g_{0}-g_{s}}{s}\right)+\frac{2-s}{2} \Phi\left(\frac{g_{s}-g_{2}}{2-s}\right) \text { almost everywhere. }
$$


In an analogous manner, it follows that

$$
\Phi\left(g_{s}\right)=\frac{s}{2} \Phi\left(\frac{g_{s}-g_{0}}{s}\right)+\frac{2-s}{2} \Phi\left(\frac{g_{s}-g_{2}}{2-s}\right) \text { almost everywhere. }
$$

Since $\Phi$ is even, the right-hand sides of (1) and (2) are equal and hence $\Phi\left(g_{s}\right)=$ $\Phi\left(g_{0}\right)$ almost everywhere. From this and the definitions of $x_{0}$ and $D$, it follows that

$$
\left|g_{s}\right|=\left|g_{0}\right| \text { almost everywhere on } D \text {. }
$$

Let $n$ be a positive integer. For each $i=0,1, \ldots, 2 n-1$, define

$$
D_{i}=\left\{\omega \in D: g_{0}(\omega)=g_{i / n}(\omega)=-g_{(i+1) / n}(\omega)\right\} \text {. }
$$

By applying (3) with $s=m / n$ for $m=1, \ldots, 2 n-1$, it follows that $D=$ $\cup_{i=0}^{2 n-1} D_{i}$ where equality holds up to a $\mu$-null set. So there is a $j$ in $\{0,1, \ldots, 2 n$ - 1) such that $\mu\left(D_{j}\right)>0$. Define a girth curve $h:[0,4] \rightarrow L^{\Phi}(\mu)$ by $h_{s}=$ $g_{(j / n)+s(\bmod 4)}$ for $s$ in [0,4]. Apply (1) to $h$ with $s=1 / n$ to obtain a point $\omega$ in $D_{j}$ such that

$$
\begin{aligned}
\Phi\left(g_{j / n}(\omega)\right)= & \frac{1}{2 n} \Phi\left(n\left(g_{j / n}(\omega)-g_{(j+1) / n}(\omega)\right)\right) \\
& +\frac{2 n-1}{2 n} \Phi\left(\frac{n}{2 n-1}\left(g_{(j+1) / n}(\omega)+g_{j / n}(\omega)\right)\right) .
\end{aligned}
$$

But $\omega$ is in $D_{j}$ and so (4) reduces to $2 n \Phi\left(g_{0}(\omega)\right)=\Phi\left(2 n g_{0}(\omega)\right)$. This implies $\Phi$ is linear on the interval $\left[0,2 n\left|g_{0}(\omega)\right|\right]$ and hence, since $\omega$ is in $D$, on the interval $\left[0,2 n x_{0}\right]$.

Since $x_{0}>0$ and $n$ was arbitrary, it follows that $\Phi(x)=k|x|$ for some $k>0$ and the proof is complete.

\section{REFERENCES}

1. R. E. Harrell and L. A. Karlovitz, Girths and flat Banach spaces, Bull. Amer. Math. Soc. 76 (1970), 1288-1291.

2.

Nonreflexivity and girth of spheres (Proc. Third Sympos. Inequalities, U.C.L.A., 1969), Inequalities, vol. III, Academic Press, New York, 1972, pp. 121-127.

3. M. A. Krasnosel'skii and Ya. B. Rutickii, Convex functions and Orlicz spaces, Noordhoff, Groningen, 1961.

4. P. Nyikos and J. J. Schäffer, Flat spaces of continuous functions, Studia Math. 42 (1972), 221-229.

5. J. J. Schäffer, Geometry of spheres in normed spaces, Lecture Notes in Pure and Appl. Math., vol. 20, Dekker, New York and Basel, 1976.

6. J. J. Schäffer, On the geometry of spheres in L-spaces, Israel J. Math. 10 (1971), 114-120.

7. B. Turett, Rotundity of Orlicz spaces, Nederl. Akad. Wetensch. Proc. Ser. A $79=$ Indag. Math. 38 (1976), 462-469.

MATHematisch InstituUt, Univiersiteit van Amsterdam, Roetersstrant 15, 1018 WB AmsterDAM, THE NeTHERLANDS

Department of Mathematics and Statistics, Miami Universtry, Oxpord, Ohio 45056

Department of Mathematical Sciences, OAkland University, Rochester, Michigan 48063 